\title{
Clinical implications of pathological features of primary membranous nephropathy
}

\author{
Xiao-dan Zhang ${ }^{1}$, Zhao Cui ${ }^{1 *}$, Mu-fan Zhang ${ }^{1}$, Jia Wang ${ }^{1}$, Yi-miao Zhang ${ }^{1}$, Zhen Qu', Xin Wang ${ }^{1}$, Jing Huang ${ }^{1}$, \\ Fang Wang ${ }^{1}$, Li-qiang Meng ${ }^{1}$, Xu-yang Cheng ${ }^{1}$, Su-xia Wang ${ }^{2}$, Gang Liu ${ }^{1}$ and Ming-hui Zhao ${ }^{1,3}$
}

\begin{abstract}
Background: The clinical outcome varies considerably in primary membranous nephropathy (pMN). Risk factors for kidney prognosis include ageing, male gender, persistent heavy proteinuria, decreased eGFR at presentation, persistent elevation of anti-PLA2R antibodies, no remission, and so on. It was controversial whether the histopathological features of pMN could predict treatment response and kidney outcome.

Methods: A retrospective study was conducted in 371 patients with biopsy-proven pMN. Pathological parameters included immunofluorescence staining, membranous Churg's stages, sclerosis, crescent, focal segmental sclerosis lesion, chronic and acute tubulointerstitial injury. The fluorescence intensity was determined: 0 , negative; 1 , weak; 2 , moderate; 3 , strong; 4, glaring. Chronic tubulointerstitial injury was graded by the involved area: 0, 0-5\%; 1, 6-25\%; 2, 26-50\%; 3, > 50\%.

Results: We found that patients with higher intensity of C3 staining, advanced membranous stage, and more severe chronic tubulointerstitial injury presented with higher positivity rate of anti-PLA2R antibodies, higher levels of urinary protein excretion and serum creatinine, and lower level of serum albumin. Univariate Cox regression analysis showed that severe (grade $=3)$ chronic tubulointerstitial injury was a risk factor to the kidney outcome of ESKD $(\mathrm{HR}=61.02,95 \% \mathrm{Cl}, 7$. $75-480.57, P<0.001)$ and over 50\% reduction of eGFR ( $H R=4.43,95 \% \mathrm{Cl}, 1.26-15.6, P=0.021)$. Multivariate analysis demonstrated it as an independent risk factor to ESKD (HR=25.77, 95\% Cl, 1.27-523.91, $P=0.035)$. None of the pathological parameters exerted any influence on treatment response $(P>0.05)$.

Conclusions: We found the prognostic role of chronic tubulointerstitial injury to the kidney outcome of pMN. This study highlighted the value of kidney biopsy under the widespread usage of anti-PLA2R antibodies for diagnosis and prognosis.
\end{abstract}

Keywords: Membranous nephropathy, Pathology, Tubulointerstitial injury, Prognosis

\section{Background}

Primary membranous nephropathy (pMN) is the most common cause of adult-onset nephrotic syndrome [1]. The diagnosis of $\mathrm{pMN}$ depends on the pathological manifestation which is characterized by the presence of subepithelial immune complexes, diffuse thickening of glomerular basement membrane by light microscopy, and granular deposit of IgG and complement along the periphery of glomerular capillary loops by immunofluorescence. M-type

\footnotetext{
* Correspondence: cuizhao@bjmu.edu.cn

'Renal Division, Key Laboratory of Renal Disease, Ministry of Health of China, Key Laboratory of CKD Prevention and Treatment, Ministry of Education of China, Peking University First Hospital, Institute of Nephrology, Peking University, Beijing 100034, People's Republic of China

Full list of author information is available at the end of the article
}

phospholipase A2 receptor (PLA2R), a transmembrane protein expressed on podocytes, has been defined as the major autoantigen of this disease [2]. The specific antigen is co-localized with IgG4 in the subepithelial immune deposits. Circulating antibodies against PLA2R were detected in about $70 \%$ of patients. It has been documented that the presence of anti-PLA2R has close associations with clinical parameters, thus the antibody has been utilized as a good biomarker in clinical practice [2].

The clinical consequence of pMN varies considerably. If untreated, about one-third of patients undergo spontaneous remission [3], especially those with absent or low levels of anti-PLA2R antibodies [4], another one-third of patients progress to end-stage kidney disease (ESKD) over

(C) The Author(s). 2018 Open Access This article is distributed under the terms of the Creative Commons Attribution 4.0 International License (http://creativecommons.org/licenses/by/4.0/), which permits unrestricted use, distribution, and 
10 years [5], and the remainder develop non-progressive chronic kidney disease (CKD). However, if with proper management, only $10 \%$ or less will develop ESKD over the subsequent 10 years [6].

Various risk factors affect kidney prognosis. The established ones include age, male gender, persistent heavy proteinuria, decreased GFR on presentation, increased excretion of $\beta 2$ microglobulin and increased urinary excretion of C3dg and C5b-9 [3, 7-12]. Persistent elevation of anti-PLA2R levels after therapy is a significant indicator to poor prognosis $[9,10]$. However, the findings on histologic variables are controversial. The advanced stage of glomerular lesions detected by electron microscopy has been suggested to correlate with worse renal survival in some but not all studies [13]. Several researches have reported that chronic interstitial fibrosis and tubular atrophy are independent predictors of progressive renal failure [14]. In addition, focal segmental glomerular sclerosis superimposed on MN has been found as indicator of a poor prognosis [15], while its importance was not substantiated in a retrospective analysis of a large cohort from the University of Toronto [16].

Using a large cohort of patients with biopsy-proven pMN, we have demonstrated that the presence of crescents showed undesirable response to the treatments and worse kidney outcomes [17]. In the current study, we investigated the histopathologic features of pMN patients with credible medical records of therapeutic responses and kidney outcomes during follow-up, aiming to clarify the clinical indications of pathological parameters of pMN.

\section{Methods}

\section{Study population}

A total of 371 consecutive patients with kidney biopsy-proven $\mathrm{pMN}$, who were diagnosed and followed up in Peking University First Hospital from 2008 to 2016, were reviewed retrospectively in this study. Patients with known secondary $\mathrm{MN}$, such as hepatitis $\mathrm{B} / \mathrm{C}$ virus infection, lupus, malignancy, rheumatoid arthritis, medications, and heavy metal poisoning, were excluded. Clinical data were collected from medical records at the time of diagnosis as well as during follow-up.

The research was in compliance of the Declaration of Helsinki and was approved by the ethics committee of Peking University First Hospital. Written informed consent was obtained for sampling tissue and blood.

Estimated glomerular filtration rate (eGFR) was calculated from serum creatinine levels using the Modification of Diet in Renal Disease Study equation adjusted for Chinese populations: eGFR $=175 \times(\text { serum creatinine })^{1.234} \times$ age $^{0.179} \times 0.79$ (if female) [18].

\section{Kidney biopsies}

Kidney biopsy was performed at the time of diagnosis in all patients. Renal specimens were evaluated with direct immunofluorescence, light microscopy, and electron microscopy, as described previously [19]. The methods were performed according to the standard operating procedure at our center [19]. Direct immunofluorescence for IgG subclasses was performed after 2014, using mouse anti-IgG1, -IgG2, -IgG3 and -IgG4 monoclonal antibodies (clone no. 4E3, HP6014, HP6050, HP6025; Southern Biotech, Birmingham, AL) at 1:100, as reported previously [20].

The fluorescence intensity of IgG, IgM, IgA, C3, C1q, and IgG subclass was determined using a semi-quantitative scale of 0 to 4: 0 , negative; 1 , weak staining; 2 , moderate staining; 3 , strong staining; 4 , glaring staining.

Glomerular MN lesions were classified into four stages according to the Ehrenreich and Churg's classification criteria. The chronic tubulointerstitial injury was defined as tubular atrophy and interstitial fibrosis, while the acute tubulointerstitial injury included the loss of tubular brush border and interstitial mononuclear cell infiltration. The scoring was graded semi-quantitatively from 0 to $4: 0,0$ $5 \% ; 1,6-25 \%$ of interstitium involved; $2,26-50 \%$ of interstitium involved; $3,>50 \%$ of interstitium involved.

\section{Detection of circulating anti-PLA2R antibodies and anti- THSD7A antibodies}

Circulating anti-PLA2R antibodies and circulating anti-thrombospondin type 1 domain containing 7A (THSD7A) antibodies were detected using commercial ELISA kits (EUROIMMUN AG, Lübeck, Germany) and immunofluorescence assay kit (EUROIMMUN AG, Lübeck, Germany) separately, following the standard instructions $[17,21]$.

\section{Treatment and follow-up}

The use of corticosteroids and immunosuppressive agents, and the definitions of remission and relapse were in compliance with the 2012 KDIGO (Kidney Disease: Improving Global Outcomes) guideline for glomerulonephritis [22].

For evaluation of the renal outcomes, the primary endpoint was ESKD; the second endpoint was renal dysfunction, defined as eGFR reduction by more than $50 \%$ from the baseline (at the time of kidney biopsy) and the final eGFR being less than $60 \mathrm{ml} / \mathrm{min} / 1.73 \mathrm{~m}^{2}$.

\section{Toronto risk score}

Toronto Risk Score by Cattran, et al. [23] was applied in this study. Toronto Risk Score $=\mathrm{e}^{\mathrm{x}} /\left(1+\mathrm{e}^{\mathrm{x}}\right), \mathrm{x}=1.26+0.3^{*}$ persistent proteinuria- $0.3 *$ slope creatinine clearance- $0.05 \%$ initial creatinine clearance; creatinine clearance was presented as eGFR; initial creatinine clearance was collected at baseline; the slope of creatinine clearance was calculated during the first 6 months. Persistent proteinuria was the minimum value of 24-h urinary protein excretion in the first six-month. [23, 24] 


\section{Statistical analysis}

Statistical analysis was performed using the SPSS statistical software package, version 13.0 (SPSS Inc., Chicago, IL). Normally distributed variates were expressed as the mean $\pm \mathrm{SD}$, and continuous variates of unnormal distribution were expressed as the median with interquartile range (IQR). Categorical variables were expressed as absolute values and percentages. For continuous variates, comparisons between two groups were performed using Student's $t$ test for normally distributed data and Wilcoxon rank-sum test for nonparametric data; comparisons among three or more groups were performed using one-way analysis of variance (ANOVA) for normally distributed data and Kruskal-Wallis test for nonparametric data. For categorical variables, Chi-square, Fisher exact test or Pearson Chi-square test were performed appropriately. If the differences among multi-groups were significant, SNK or Dunnett's test was used to analyze differences between each pair of groups. Risk factors for no-remission after treatments were analyzed using Logistic regression model. Kaplan-Meier curves were used to analyze the kidney outcomes of patients. The predictors for kidney dysfunction were assessed using Cox regression model. Statistically significant parameters $(P<0.10)$ were enrolled into the Multivariate Cox regression models. Results were expressed as odds ratio (OR) or hazard ratio (HR) with $95 \%$ confidence interval (CI). All statistical analyses data were 2-tailed and the level of significance was set at 0.05 .

\section{Results}

\section{Clinical and pathological data}

Three hundred seventy-one consecutive patients with kidney biopsy-proven pMN were reviewed in this study. The clinical and pathological data are shown in Table 1.

Three hundred sixty seven (98.9\%) patients presented with granular staining of IgG and 351 (94.6\%) patients showed C3 staining along the glomerular capillary walls (Table 1). They all had immune deposit on the epithelial area by electron microscopy. IgG subclasses staining was available in 171 consecutive patients after 2014. Among them, IgG4 deposit was shown in 167 (97.7\%) patients and IgG4 dominance was in 154 (90.1\%) patients.

The stage of membranous lesion was parallel with the intensity of C3 deposition $(r=0.147, P=0.005)$ and the grades of chronic tubulointerstitial injury $(r=0.247, P<$ $0.001)$. There were 48 (12.9\%) patients having global sclerosis with a median percentage of $5.1(3.0-7.4) \%$, and 19 (5.1\%) patients having focal segmental sclerosis with a median percentage of $5.3(4.2-6.3) \%$. Crescents were shown in $29(7.8 \%)$ patients with a median percentage of $4.2(2.7-$ $5.1) \%$. The global sclerosis $(7.0 \%$ vs. $7.1 \%$ vs. $17.4 \%$ vs. $12.5 \%, P=0.030)$ and crescent $(1.0 \%$ vs. $7.1 \%$ vs. $11.1 \%$ vs. $12.5 \%, P=0.005)$ were more frequently shown in the patients with severer chronic tubulointerstitial injury (Fig. 1).

\section{The associations between pathological and clinical features}

According to the increase of C3 staining intensity (Table 2), the patients presented with higher positivity rate of serum anti-PLA2R antibodies, higher level of urinary protein excretion, lower level of serum albumin, and higher level of serum creatinine. There was no difference on clinical features among the patients with different intensity of IgG staining $(P>0.05)$. The patients with IgG4 subclass dominant presented with higher level of eGFR [115.3 (97.8-146.2) vs. $92.0(73.1-126.4) \mathrm{ml} /$ $\left.\min / 1.73 \mathrm{~m}^{2}, P<0.001\right]$.

With the aggravation of $\mathrm{MN}$ stages from I to III (Additional file 1: Table S1), the patients presented with higher positivity rate of serum anti-PLA2R antibodies, higher level of urinary protein excretion, and lower level of eGFR.

With the deterioration of tubulointerstitial injury from grade 0 to 3 (Table 3), the patients became older, and presented with higher positivity rate of serum anti-PLA2R antibodies, higher level of urinary protein excretion, lower level of serum albumin, lower level of eGFR, higher percentage of hypertension, and lower level of hemoglobin.

The 32 patients with acute tubulointerstitial injury (Additional file 2: Table S2) were older and presented with higher level of urinary protein excretion, lower level of serum albumin, lower level of eGFR, higher percentage of hypertension, and lower level of hemoglobin.

We found no correlation between anti-PLA2R antibody level and pathological features $(P>0.05)$.

\section{Treatment responses}

Among the 371 patients, 254 (68.5\%) patients were treated by immunosuppressive drugs and the others by ACEI/ ABRs only. $325(87.6 \%)$ patients achieved remission, including 168 (45.3\%) patients of complete remission and 157 (42.3\%) patients of partial remission. Nephrotic relapse occurred in 90/325 (27.7\%) patients (Table 1).

Univariate Logistic regression analysis showed that the higher level of proteinuria $(\mathrm{OR}=1.08,95 \% \mathrm{CI} 1.02-1.15$, $P=0.016$ ), the positivity of serum anti-PLA2R antibodies $(\mathrm{OR}=4.77,95 \% \mathrm{CI} 1.83-12.41, P=0.001)$, and the higher level of serum anti-PLA2R antibodies $(\mathrm{OR}=1.05,95 \% \mathrm{CI}$ $1.02-1.09, P=0.002)$ were risk factors to the no-remission response after treatments. Multivariate Logistic regression analysis showed that the positivity of serum anti-PLA2R antibodies $(\mathrm{OR}=3.20,95 \% \mathrm{CI} 1.18-8.72, P=0.023)$ was an independent risk factors to no-remission (Additional file 3: Table S3). Clinical risk factors were evaluated by Toronto risk score. After adjusting by Toronto risk score, the positivity of anti-PLA2R antibodies $(\mathrm{OR}=4.63,95 \% \mathrm{CI} 1.56-$ 13.70, $P=0.006)$ was still an independent risk factor to no-remission.

All the pathological features did not exert any influence on the treatment response of pMN $(P>0.05)$. 
Table 1 Clinical characters of the patients with pMN

\begin{tabular}{|c|c|}
\hline & $N=371$ \\
\hline Age (years) & $53,43-61$ \\
\hline Gender (M/F) & $207 / 164$ \\
\hline Nephrotic syndrome, n (\%) & $255(68.7 \%)$ \\
\hline Proteinuria (g/24 h) & $4.0,2.3-6.4$ \\
\hline Serum albumin $(\mathrm{g} / \mathrm{L})$ & $27.5 \pm 6.1$ \\
\hline Hematuria, n (\%) & $208(56.1 \%)$ \\
\hline Hypertension, n (\%) & $187(50.4 \%)$ \\
\hline Serum creatinine $(\mu \mathrm{mol} / \mathrm{L})$ & $65.4,54.0-80.0$ \\
\hline eGFR $\left(\mathrm{ml} / \mathrm{min}\right.$ per $\left.1.73 \mathrm{~m}^{2}\right)$ & $114.2,92.6-143.2$ \\
\hline Anti-PLA2R antibody positivity, $\mathrm{n}(\%)$ & $243(65.5 \%)$ \\
\hline Anti-PLA2R antibody level ${ }^{\mathrm{a}}(\mathrm{U} / \mathrm{mL})$ & $77.8,34.9-200.0$ \\
\hline Anti-THSD7A antibody positivity, n (\%) & $4 / 295(1.4 \%)$ \\
\hline
\end{tabular}

Pathological features

Immunofluorescence

$$
\begin{aligned}
& \text { IgG deposit, n (\%) } \\
& \operatorname{lgG} 1, \mathrm{n}(\%) \\
& \operatorname{lgG} 2, \mathrm{n}(\%) \\
& \operatorname{lgG} 3, \mathrm{n}(\%) \\
& \operatorname{lgG} 4, \mathrm{n}(\%) \\
& \text { IgA deposit, n (\%) } \\
& \text { IgM deposit, n (\%) } \\
& \text { C3 deposit, n (\%) } \\
& \text { C1 q deposit, n (\%) }
\end{aligned}
$$

367 (98.9\%)

$151 / 171(88.3 \%)$

150/171 (87.7\%)

126/171 (73.7\%)

167/171 (97.7\%)

$103(27.8 \%)$

161 (43.4\%)

$351(94.6 \%)$

$151(40.7 \%)$

Glomerular lesion

$$
\begin{aligned}
& \text { Stage I, n (\%) } \\
& \text { Stage II, n (\%) } \\
& \text { Stage III, n (\%) } \\
& \text { Stage IV, n (\%) }
\end{aligned}
$$

Global sclerosis (\%)

Crescent (\%)

Focal segmental glomerular sclerosis (\%)

Chronic tubulointerstitial injury

$$
\begin{aligned}
& \text { Grade }=0 \\
& \text { Grade }=1 \\
& \text { Grade }=2 \\
& \text { Grade }=3
\end{aligned}
$$

$193(52.0 \%)$

$159(42.9 \%)$

$19(5.1 \%)$

$0(0 \%)$

$5.1,3.0-7.4$

$4.2,2.7-5.1$

$5.3,4.2-6.3$

$100(27.0 \%)$

$56(15.1 \%)$

$207(55.8 \%)$

$8(2.1 \%)$

$32(8.6 \%)$

Acute tubulointerstitial injury

Treatments

ACEl/ARBs only, $n$ (\%)

Immunosuppressive therapies, $\mathrm{n}(\%)$

Cyclophosphamide + corticosteroids

Calcineurin inhibitor w/o corticosteroids

Rituximab
Table 1 Clinical characters of the patients with pMN (Continued)

\begin{tabular}{ll}
\hline & $N=371$ \\
\hline Treatment responses & \\
Complete remission, $\mathrm{n}(\%)$ & $168(45.3 \%)$ \\
Partial remission, $\mathrm{n}(\%)$ & $157(42.3 \%)$ \\
$\quad$ No remission, $\mathrm{n}(\%)$ & $46(12.4 \%)$ \\
Relapse, $\mathrm{n}(\%)$ & $90 / 325(27.7 \%)$ \\
Follow-up duration (months) & $26.9,13.9-45.4$ \\
ESKD, $\mathrm{n}(\%)$ & $8(2.2 \%)$ \\
eGFR reduction > 50\% from baseline, $\mathrm{n}(\%)$ & $67(18.1 \%)$ \\
\hline
\end{tabular}

${ }^{a}$ Antibody level was calculated only for those over the cut-off value of $20 \mathrm{U} /$ $\mathrm{mL}$. Continuous and normally distributed variables were presented as mean \pm $\mathrm{SD}$; continuous and non-normally distributed variables were presented as median, IQR; categorical variables were presented as number (\%)

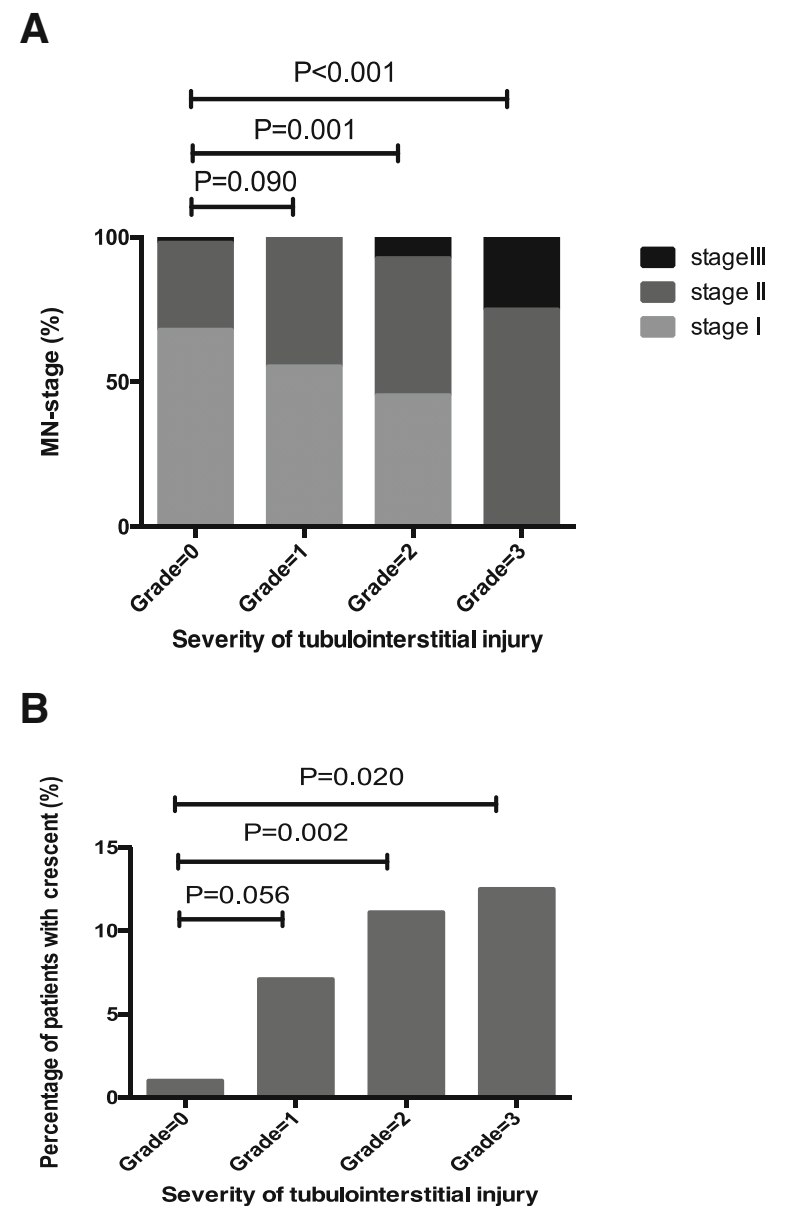

Fig. 1 The comparison of pathological parameters in the pMN patients with different severity of tubulointerstitial injury. With the deterioration of tubulointerstitial injury, the stage of membranous lesion became more advanced (a) and the prevalence of crescent formation was increasing (b) 
Table 2 Comparisons of clinical characteristics of pMN patients with different intensity of C3 deposit

\begin{tabular}{|c|c|c|c|c|c|}
\hline & C3 $-(n=20)$ & C3 $1+(n=114)$ & C3 $2+(n=186)$ & $\mathrm{C} 33+(n=51)$ & $P$ \\
\hline Age (year) & $53(48-60)$ & $53(40-60)$ & $53(43-62)$ & $52(47-61)$ & 0.822 \\
\hline Gender (M/F) & $11 / 9$ & $61 / 53$ & $98 / 88$ & $37 / 14$ & 0.079 \\
\hline Nephrotic syndrome, n (\%) & $11(55.0 \%)$ & $67(58.8 \%)$ & $133(71.5 \%)$ & $44(86.3 \%)$ & 0.002 \\
\hline Proteinuria (g/24 h) & $3.6(2.3-7.1)$ & $3.4(2.3-5.8)$ & $4.0(2.2-6.0)$ & $6.2(3.5-9.6)$ & 0.001 \\
\hline Serum albumin ( $g / L)$ & $30.1 \pm 8.7$ & $29.7 \pm 5.9$ & $26.7 \pm 5.5$ & $24.4 \pm 5.7$ & $<0.001$ \\
\hline Microscopic hematuria, n (\%) & $4(20.0 \%)$ & $59(52.7 \%)$ & $112(60.5 \%)$ & $33(66.0 \%)$ & 0.002 \\
\hline Serum creatinine $(\mu \mathrm{mol} / \mathrm{L})$ & $62.5(50.0-80.8)$ & $62(53.7-79.3)$ & $66.0(53.0-79.5)$ & $71.0(62.6-93.4)$ & 0.048 \\
\hline eGFR $\left(\mathrm{ml} / \mathrm{min}\right.$ per $\left.1.73 \mathrm{~m}^{2}\right)$ & $120.0(99.2-143.4)$ & 116.9(99.9-151.2) & $115.2(90.5-142.5)$ & 102.6(80.4-127.0) & 0.071 \\
\hline Anti-PLA2R antibody positivity, n (\%) & $11(55.0 \%)$ & $58(51.3 \%)$ & $136(73.5 \%)$ & $38(74.5 \%)$ & $<0.001$ \\
\hline Anti-PLA2R antibody level (U/mL) & $76.3(58.2-160.7)$ & $97.5(52.6-186.2)$ & $117.8(50.8-235.7)$ & $135.3(45.0-277.1)$ & 0.653 \\
\hline Anti-THSD7A antibody positivity, n(\%) & $0(0.0 \%)$ & $2(2.2 \%)$ & $2(1.3 \%)$ & $0(0.0 \%)$ & 0.741 \\
\hline Hypertension, n (\%) & $8(40.0 \%)$ & $62(54.4 \%)$ & $88(47.3 \%)$ & $29(56.9 \%)$ & 0.369 \\
\hline Hemoglobin (g/L) & $131.0(119.0-144.0)$ & $137.5(128.0-151.0)$ & $137.0(126.0-149.0)$ & $133.0(115.3-143.5)$ & 0.059 \\
\hline
\end{tabular}

Continuous and normally distributed variables were presented as mean $\pm \mathrm{SD}$; continuous and non-normally distributed variables were presented as median, IQR; categorical variables were presented as number (\%)

The numbers in boldface are statistically significant

\section{Kidney outcomes}

During a median follow up of 26.9 (13.9-45.4) months, 8/371 (2.2\%) patients progressed to ESKD, 67 (18.1\%) patients experienced kidney dysfunction with an over $50 \%$ decline of eGFR from the baseline at kidney biopsy.

Kaplan-Meier curve showed that the kidney outcome of ESKD $(P<0.001)$ (Fig. 2) and kidney dysfunction $(P=0.079)$ became worse according to the deterioration of tubulointerstitial injury.

Univariate Cox regression analysis showed that the severity of chronic tubulointerstitial injury $(\mathrm{HR}=61.02$, 95\%CI 7.75-480.57, $P<0.001$ ), acute tubulointerstitial injury $(\mathrm{HR}=6.94,95 \% \mathrm{CI} 1.65-29.22, P=0.008)$, and no-remission after treatments $(\mathrm{HR}=14.34,95 \% \mathrm{CI} 2.98-$ 68.97, $P=0.001)$ were risk factors of ESKD. The higher level of eGFR at baseline on biopsy $(\mathrm{HR}=0.97,95 \% \mathrm{CI}$ $0.95-0.99, P=0.006)$ was protective factor of ESKD. Multivariate analysis identified that the severity of chronic tubulointerstitial injury $(\mathrm{HR}=25.77,95 \% \mathrm{CI}$ 1.27-523.91, $P=0.035)$ and no-remission $(\mathrm{HR}=10.17$, 95\%CI 1.75-59.02, $P=0.010)$ were two independent risk factors of the kidney outcome ESKD (Table 4).

When the clinical risk factors were evaluated by Toronto risk score and enrolled into multivariate Cox

Table 3 Comparisons of clinical characteristics of pMN patients with different severity of chronic tubulointerstitial injury

\begin{tabular}{|c|c|c|c|c|c|}
\hline & Grade $=0(n=100)$ & Grade $=1(n=56)$ & Grade $=2(n=207)$ & Grade $=3(n=8)$ & $P$ \\
\hline Age (year) & $47(32-58)$ & $54(44-62)$ & $56(47-63)$ & $58(50-70)$ & $<0.001$ \\
\hline Gender (M/F) & $49 / 51$ & $34 / 22$ & $121 / 86$ & $3 / 5$ & 0.252 \\
\hline Nephrotic syndrome, n (\%) & $51(51.0 \%)$ & $37(66.1 \%)$ & $161(77.8 \%)$ & $6(75.0 \%)$ & $<0.001$ \\
\hline Proteinuria (g/24 h) & $2.8(1.8-4.8)$ & $3.8(2.0-6.3)$ & $4.8(2.9-7.3)$ & $7.6(4.0-11.1)$ & $<0.001$ \\
\hline Serum albumin (g/L) & $29.6 \pm 5.7$ & $29.3 \pm 7.0$ & $26.0 \pm 5.7$ & $26.3 \pm 4.8$ & $<0.001$ \\
\hline Microscopic hematuria, n (\%) & $50(50.0 \%)$ & $36(64.3 \%)$ & $118(57.6 \%)$ & $4(50.0 \%)$ & 0.422 \\
\hline Serum creatinine $(\mu \mathrm{mol} / \mathrm{L})$ & $61.3(50.9-72.9)$ & $66.2(52.8-78.9)$ & $67.3(55.0-83.0)$ & $133.4(99.5-174.6)$ & $<0.001$ \\
\hline eGFR $\left(\mathrm{ml} / \mathrm{min}\right.$ per $\left.1.73 \mathrm{~m}^{2}\right)$ & $123.1(105.4-151.5)$ & $115.5(96.9-146.9)$ & $109.8(85.8-139.5)$ & $49.1(29.3-70.5)$ & $<0.001$ \\
\hline Anti-PLA2R antibody positivity, n (\%) & $54(54.0 \%)$ & $36(64.3 \%)$ & $147(71.4 \%)$ & $6(85.7 \%)$ & 0.016 \\
\hline Anti-PLA2R antibody level (U/mL) & $105.9(46.5-195.2)$ & $106.4(61.6-181.8)$ & $117.8(51.8-241.1)$ & $299.3(40.6-818.90)$ & 0.659 \\
\hline Anti-THSD7A antibody positivity, n (\%) & $2(2.6 \%)$ & $0(0.0 \%)$ & $2(1.2 \%)$ & $0(0.0 \%)$ & 0.657 \\
\hline Hypertension, n (\%) & $33(33.0 \%)$ & $24(42.9 \%)$ & $123(59.4 \%)$ & $7(87.5 \%)$ & $<0.001$ \\
\hline Hemoglobin (g/L) & $139.0(128.0-152.0)$ & $137.0(130.0-150.0)$ & $136.0(123.0-147.0)$ & $107.0(120.8-131.5)$ & 0.004 \\
\hline
\end{tabular}

Continuous and normally distributed variables were presented as mean \pm SD; continuous and non-normally distributed variables were presented as median, IQR; categorical variables were presented as number (\%)

The numbers in boldface are statistically significant 


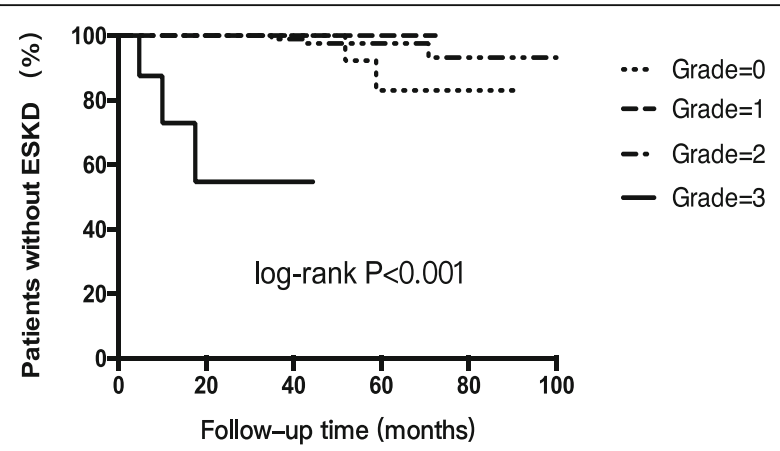

Fig. 2 Kaplan-Meier curves analysis for the end stage kidney disease (ESKD) in patients with pMN, with a comparison among the patients with different severity of chronic tubulointerstitial injury. The patients with severe tubulointerstitial injury (grade 3 ) had worse kidney outcome during follow-up

regression model together with the severity of chronic tubulointerstitial injury, none of them showed independent prognostic value to ESKD $(P>0.05)$. We found that according to the deterioration of tubulointerstitial injury from grade 0 to 3 , the patients presented with higher risk score $[0.01(0.00-0.03)$ vs. $0.01(0.00-0.08)$ vs. 0.02 $(0.00-0.30)$ vs. $0.67(0.29-0.95), P<0.001]$. The correlation $(r=0.262, P<0.001)$ between Toronto risk score and tubulointerstitial injury made the model unstable.

For the kidney dysfunction outcome, univariate Cox regression analysis found that the severity of tubulointerstitial injury $(\mathrm{HR}=4.43,95 \% \mathrm{CI} 1.26-15.59, P=0.021)$, the older age $(\mathrm{HR}=1.02,95 \% \mathrm{CI} 1.00-1.04, P=0.027)$, the positivity of serum anti-PLA2R antibodies $(\mathrm{HR}=$ 3.19 , 95\% CI 1.62-6.25, $P=0.001$ ), the level of serum anti-PLA2R antibodies $(\mathrm{HR}=1.04,95 \% \mathrm{CI} 1.02-1.06$, $P<0.001)$, and no-remission after treatments $(\mathrm{HR}=$ 3.86, 95\%CI 1.89-7.88, $P<0.001$ ) were risk factors of the eGFR reduction over $50 \%$. Toronto risk score was not a risk factor to the eGFR reduction over $50 \%(\mathrm{HR}=$ $1.01,95 \% \mathrm{CI} 0.21-4.96, P=0.987)$. Multivariate regression analysis identified that the positivity of anti-PLA2R antibodies $(\mathrm{HR}=2.54,95 \% \mathrm{CI} 1.26-5.14, P=0.009)$ and no remission $(\mathrm{HR}=3.16,95 \% \mathrm{CI} 1.46-6.86, P=0.004)$ were independent risk factors to kidney dysfunction.

\section{Discussion}

In this large consecutive cohort of patients with biopsy-proven $\mathrm{pMN}$, we performed a comprehensive investigation on the pathological parameters of pMN to explore their clinical applications. The intensity of $\mathrm{C} 3$ deposit, the stage of membranous lesion, and the severity of tubulointerstitial injury were all associated with the severity of proteinuria and the level of eGFR at the time of kidney biopsy. Only the severity of chronic tubulointerstitial injury was an independent risk factor to the kidney outcome of ESKD and was risk to kidney dysfunction defined as eGFR reduction over $50 \%$. None of the pathological parameters was associated with treatment responses.

We found that the intensity of C3 deposit, the stage of membranous lesion, and the severity of tubulointerstitial injury were all related to the positivity of anti-PLA2R antibodies. This finding implies that the autoantibody was not only a biomarker for clinical presentation of pMN but also a good indicator for the pathological features. There was a correlation between the granular staining of $\mathrm{C} 3$ and the proteinuria and kidney function of the patient, which was not observed of IgG deposit. It implies that the deposited anti-PLA2R-IgG may be the beginning of autoimmune disorder and the subsequent complement activation might be more effective to induce kidney pathological injury.

There are many studies focus on the prognostic significance of histopathological parameters of pMN, but a large diversity exists $[16,25]$. The reason may be that different parameters were assessed in different studies. In the current study, we investigated all the pathological parameters including immunofluorescence intensity, membranous stages, acute and chronic tubulointerstitial injury, crescent formation, global and focal segmental sclerosis, together with the clinical features including anti-PLA2R antibodies and different treatment regiments and responses, in a large cohort of pMN patients. Then we found that the severity of chronic tubulointerstitial injury was an independent risk factor to the kidney outcome of ESKD. It was also a risk factor to kidney dysfunction. These results were consistent with previous studies [16, 26-31]. Although a Parisian study showed that interstitial fibrosis was not significantly associated with cumulative renal insufficiency [32], when FSGS was removed as a variable, they declared that the interstitial fibrosis showed a significant association with renal insufficiency. In the histopathological report of pMN kidney biopsy, we highly recommend to describe the grades of chronic tubulointerstitial injury, instead of membranous lesion alone. It could provide helpful information to the physicians on kidney prognosis.

We found that severe ( $>50 \%$ of interstitium involved) chronic tubulointerstitial injury was a risk factor to ESKD. It is also suggested by another Chinese study [28]. However, Chen et al. found that the moderate or severe damage $(>25 \%$ of interstitium involved) was risk factor to ESKD [29]. The possible reason for this discrepancy may be that the patients in Chen's study were restricted to those with nephrotic-range proteinuria (>3.5 g/day) and CKD stages 2-4. In that ESKD high-risk cohort, the moderate tubulointerstitial injury might show unfavorable outcomes, while in the general pMN patients, more severe tubulointerstitial injury may have risk effect to the outcome of ESKD. 
Table 4 The risk factors of ESKD in patients with pMN

\begin{tabular}{|c|c|c|c|c|}
\hline & \multicolumn{2}{|l|}{ Univariate analysis } & \multicolumn{2}{|l|}{ Multivariate analysis } \\
\hline & $\mathrm{HR}(95 \% \mathrm{Cl})$ & $P$ & $\mathrm{HR}(95 \% \mathrm{Cl})$ & $P$ \\
\hline Age (increased by 1 year) & $1.002(0.946-1.062)$ & 0.935 & & \\
\hline Gender (male) & $0.513(0.230-1.142)$ & 0.102 & & \\
\hline Proteinuria (increased by $1 \mathrm{~g} / 24 \mathrm{~h}$ ) & $1.066(0.924-1.229)$ & 0.380 & & \\
\hline Serum albumin (increased by $1 \mathrm{~g} / \mathrm{L}$ ) & $0.952(0.844-1.074)$ & 0.426 & & \\
\hline eGFR (increased by $1 \mathrm{ml} / \mathrm{min}$ per $1.73 \mathrm{~m}^{2}$ ) & $0.971(0.951-0.992)$ & 0.006 & $0.988(0.957-1.020)$ & 0.464 \\
\hline Anti-PLA2R antibody positivity & $36.361(0.043-30,631.182)$ & 0.296 & & \\
\hline Anti-PLA2R antibody level (increased by $20 \mathrm{U} / \mathrm{mL}$ ) & $0.911(0.746-1.112)$ & 0.360 & & \\
\hline Anti-THSD7A antibody positivity & 0.049 (0.000-) & 0.904 & & \\
\hline \multicolumn{5}{|l|}{ C3 staining } \\
\hline negative & ref & - & & \\
\hline $1+$ & $1.077(0.404-2.871)$ & 0.882 & & \\
\hline $2+$ & $0.991(0.387-2.535)$ & 0.984 & & \\
\hline $3+$ & $0.689(0.209-2.275)$ & 0.542 & & \\
\hline \multicolumn{5}{|l|}{ MN-stage } \\
\hline 1 & ref & - & & \\
\hline$\|$ & $3.020(0.585-15.599)$ & 0.187 & & \\
\hline III & $5.741(0.514-64.088)$ & 0.156 & & \\
\hline Global sclerosis (increased by 1\%) & $0.577(0.108-3.082)$ & 0.577 & & \\
\hline Crescent (increased by 1\%) & $0.530(0.057-4.940)$ & 0.578 & & \\
\hline Focal segmental glomerular sclerosis (increased by 1\%) & $0.644(0.048-8.623)$ & 0.740 & & \\
\hline \multicolumn{5}{|l|}{ Chronic tubulointerstitial injury } \\
\hline Grade $=0$ & ref & - & ref & - \\
\hline Grade $=1$ & $0.000(0.000-0.000)$ & 0.985 & $0.000(0.000-0.000)$ & 0.984 \\
\hline Grade $=2$ & $0.445(0.073-2.702)$ & 0.379 & $0.460(0.071-2.971)$ & 0.415 \\
\hline Grade $=3$ & $61.016(7.747-480.574)$ & $<0.001$ & $25.767(1.267-523.911)$ & 0.035 \\
\hline Acute tubulointerstitial injury & $6.941(1.649-29.217)$ & 0.008 & $0.897(0.078-9.685)$ & 0.908 \\
\hline \multicolumn{5}{|l|}{ Treatments } \\
\hline ACEI/ARBS & ref & - & & \\
\hline Cyclophosphamide with corticosteroids & $3.002(0.501-18.003)$ & 0.229 & & \\
\hline Calcineurin inhibitor w/o corticosteroids & $3.479(0.490-24.722)$ & 0.213 & & \\
\hline No remission & $14.339(2.981-68.970)$ & 0.001 & $10.161(1.749-59.018)$ & 0.010 \\
\hline
\end{tabular}

$H R$ hazard ratio, $\mathrm{Cl}$ confidence interval

The numbers in boldface are statistically significant

Another risk factor to ESKD was revealed as no-remission in the current study. The clinical composite risk score, Toronto risk score, was also calculated and enrolled in the multivariate Cox regression model. However, since there was close correlation between Toronto risk score and the severity of chronic tubulointerstitial injury, none of them showed independent risk effect to ESKD.

The mechanism remains unclear for that tubulointerstitial damage possesses such an important effect in a glomerular disease. The hypothesis of inflammation-sclerosis is well recognized. The numbers of interstitial macrophages and $\mathrm{T}$ cells and the increase of interstitial collagen IV and VI were important factors for pMN prognosis [30]. The tubular epithelial cells can be activated by the cytokines produced by the monocytes/macrophages in the glomerular inflammation lesion and can act as antigen presenting cells to induce the infiltration of $\mathrm{T}$ lymphocytes and monocytes/macrophages in the interstitia. The latter may secrete transforming growth factor beta and produce lymphokines to stimulate fibroblasts [33].

We found that none of the pathological parameters showed any effect on treatment responses. Even the patients presented with $\mathrm{C} 3$ staining $3+$, membranous stage 
III, acute or severe tubulointerstitial injury, they still had the chance to get complete or partial remission after treatments and to avoid the worse kidney outcomes consequently. This finding may encourage the physicians to give active treatments without scruple of the probably unfavorable effect from the advanced membranous lesion. Different therapies, cyclophosphamides or calcineurin inhibitors, resulted in comparable treatment responses, which was consistent with 2012 KDIGO guideline. The only independent risk factor to no-remission was revealed as the positivity of anti-PLA2R antibodies, together with the possible risk factors as proteinuria and antibody level. These results highlighted the importance of therapeutic strategy to modulate the autoimmune disorders in pMN. Rituximab should be considered for the clearance of autoantibody-producing B cells [10].

There are two advantages in our study. First, all the pathological parameters were comprehensively included into the models to explore their impacts on kidney outcomes in this large cohort, which makes the results much reliable. The second advantage is the enrollment of anti-PLA2R antibody (positivity rate and antibody level) as one of the clinical features, which has been revealed as a prognostic factor to both treatment responses and kidney outcomes [10, 34, 35]. The severity of pathological injury was related to antibody positivity. Multivariate Cox analysis showed that severe chronic tubulointerstitial injury was an independent risk factor to ESKD, but not anti-PLA2R antibody which could be depleted by immunosuppressive therapies. Anti-PLA2R antibody positivity and higher level were risk factors to no-remission. And no-remission was another independent risk factor to ESKD. Thus, anti-PLA2R antibody may display its prognostic role by affecting on treatment response. This finding highlights the necessity of kidney biopsy for pMN, despite the diagnosis based on positive anti-PLA2R antibodies. The information on tubulointerstitial injury and crescent formation could be helpful for evaluating prognosis.

There are limitations in the current study. As a retrospective study, the staining of PLA2R in glomeruli was not available, which might lead to an underestimation of the percentage of PLA2R-related MN. Another limitation was the short time of follow-up. For a slowly progressive glomerular disease like pMN, 7-10 years of follow-up is considered appropriate for the assessment of primary endpoints ESKD [6]. Thus, we added the secondary endpoint as kidney dysfunction defined as the over $50 \%$ reduction of eGFR. The unfavorable prognostic effects of tubulointerstitial injury were observed on both endpoints.

\section{Conclusions}

In conclusion, our study identified the chronic damage $>50 \%$ of interstitium as an independent predictor of kidney progression to ESKD in pMN. The grade of tubulointerstitial lesions " $\mathrm{T}$ " should be important in the histopathological report of pMN.

\section{Additional files}

Additional file 1: Table S1. Comparisons of clinical characteristics of pMN patients with different stages of membranous lesion. (DOCX $17 \mathrm{~kb}$ )

Additional file 2: Table S2. Comparisons of clinical characteristics of pMN patients with and without acute tubulointerstitial injury. (DOCX 20 kb)

Additional file 3: Table S3. The risk factors of no-remission in the patients with pMN. (DOCX $21 \mathrm{~kb})$

\section{Abbreviations}

ACEl: Angiotensin converting enzyme inhibitors; ARB: Angiotensin receptor blocker; C3: Complement 3; Cl: Confidence interval; CKD: Chronic kidney disease; eGFR: Estimated glomerular filtration rate; ESKD: End-stage kidney disease; FSGS: Focal glomerular sclerosis; HR : Hazard ratio; IQR: Interquartile range; KDIGO: Kidney Disease: Improving Global Outcomes; OR: Odds ratio; PLA2R: M-type phospholipase $A_{2}$ receptor; pMN: Primary membranous nephropathy; THSD7A : Thrombospondin type 1 domain containing 7A

\section{Acknowledgements}

The technical support by Lu Bai and Jin-ying Wang is greatly appreciated.

\section{Funding}

This work is supported by grants of Natural Science Foundation of China to the Innovation Research Group (81621092), the Outstanding Young Scholar (81622009), and other programs (81330020, 81500542), and the Capital of Clinical Characteristics and Applied Research Fund (Z161100000516039). The funders played no role in study design, data collection, analysis,

interpretation or manuscript writing.

\section{Availability of data and materials}

The present study was derived from a developing clinical cohort. Data from the cohort will not be shared currently since the whole project is not finished and the privacy of patients should be protected. If there are any requests about details of the project, please contact with the corresponding author Zhao Cui.

\section{Authors' contributions}

XDZ and ZC formed the study concept, conducted the study, analyzed the data, interpreted the results, and drafted the manuscript. MFZ, JW, YMZ, ZQ, $X W, J H, F W, L Q M, X Y C, G L$ and SXW made substantial contributions to the acquisition of data, and the analysis and interpretation of data. $\mathrm{MHZ}$ is the major sponsor, and he raised lots of important and helpful suggestions in manuscript writing and revision. Each author contributed important intellectual content during manuscript drafting or revision and agreed to be accountable for all aspects of the work. The authors have read and approved the final version of the manuscript.

Ethics approval and consent to participate

The research was in compliance of the Declaration of Helsinki and approved by the ethics committee of Peking University First Hospital. All participants

gave written contents for data collection.

Consent for publication

Not applicable.

\section{Competing interests}

The authors declare that they have no competing interests.

\section{Publisher's Note}

Springer Nature remains neutral with regard to jurisdictional claims in published maps and institutional affiliations.

\section{Author details}

${ }^{1}$ Renal Division, Key Laboratory of Renal Disease, Ministry of Health of China, Key Laboratory of CKD Prevention and Treatment, Ministry of Education of China, Peking University First Hospital, Institute of Nephrology, Peking 
University, Beijing 100034, People's Republic of China. ${ }^{2}$ Laboratory of Electron Microscopy, Pathological Centre, Peking University First Hospital, Beijing, People's Republic of China. ${ }^{3}$ Peking-Tsinghua Center for Life Sciences, Beijing 100871, PR, China.

\section{Received: 2 February 2018 Accepted: 13 August 2018}

Published online: 28 August 2018

\section{References}

1. Ronco P, Debiec H. Pathophysiological advances in membranous nephropathy: time for a shift in patient's care. Lancet. 2015 May 16; 385(9981):1983-92.

2. Beck LH Jr, Bonegio RG, Lambeau G, et al. M-type phospholipase a 2 receptor as target antigen in idiopathic membranousnephropathy. $\mathrm{N}$ Engl J Med. 2009 Jul 2;361(1):11-21.

3. Schieppati A, Mosconi L, Perna A, et al. Prognosis of untreated patients with idiopathic membranous nephropathy. N Engl J Med. 1993 Jul 8;329(2):85-9.

4. Hofstra JM, Beck LH Jr, Beck DM, et al. Anti-phospholipase A2 receptor antibodies correlate with clinical status in idiopathic membranous nephropathy. Clin J Am Soc Nephrol. 2011 Jun;6(6):1286-91.

5. Davison AM, Cameron JS, Kerr DN, et al. The natural history of renal function in untreated idiopathic membranous glomerulonephritisin adults. Clin Nephrol. 1984 Aug;22(2):61-7.

6. Ram R, Guditi S, Kaligotla VD. A 10-year follow-up of idiopathic membranous nephropathy patients on steroids and cyclophosphamide: a case series. Ren Fail. 2015 Apr;37(3):452-5.

7. Pei Y, Cattran D, Greenwood C. Predicting chronic renal insufficiency in idiopathic membranous glomerulonephritis. Kidney Int. 1992 Oct:42(4):960-6.

8. Branten AJ, du Buf-Vereijken PW, Klasen IS, et al. Urinary excretion of $\beta 2$ microglobulin and lgG predict prognosis in idiopathic membranous nephropathy: a validation study. J Am Soc Nephrol. 2005 Jan;16(1):169-74.

9. Kanigicherla D, Gummadova J, McKenzie EA, et al. Anti-PLA2R antibodies measured by ELISA predict long-term outcome in a prevalent population of patients with idiopathic membranous nephropathy. Kidney Int. 2013 May; 83(5):940-8.

10. Beck LH Jr, Fervenza FC, Beck DM, et al. Rituximab-induced depletion of anti-PLA2R autoantibodies predicts response in membranous nephropathy. J Am Soc Nephrol. 2011 Aug;22(8):1543-50.

11. Cattran DC, Kim ED, Reich H, et al. Membranous nephropathy: Quantifing remission duration on outcome. J Am Soc Nephrol. 2017 Mar;28(3):9951003.

12. Brenchley PEC, Coupes B, Short CD, et al. Urinary C3dg and C5b-9 indicate active immune disease in human membranous nephropathy. Kidney Int. 1992 Apr;41(4):933-7.

13. Ponticelli $C$, Zucchelli $P$, Imbasciati $E$, et al. Controlled trial of methylprednisolone and chlorambucil in idiopathic membranous nephropathy. N Engl J Med. 1984 Apr 12;310(15):946-50.

14. Ponticelli $C$, Zucchelli $P$, Passerini $P$, et al. A randomized trial of methylprednisolone and chlorambucil in idiopathic membranous nephropathy. N Engl J Med. 1989 Jan 5;320(1):8-13.

15. Wakai S, Magil AB. Focal glomerulosclerosis in idiopathic membranous glomerulonephritis. Kidney Int. 1992 Feb;41(2):428-34.

16. Troyanov S, Roasio L, Pandes $M$, et al. Renal pathology in idiopathic membranous nephropathy: a new perspective. Kidney Int. 2006 May;69(9): 1641-8.

17. Wang J, Zhu P, Cui Z, et al. Clinical features and outcomes in patients with membranous nephropathy and crescent formation. Medicine (Baltimore). 2015 Dec;94(50):e2294

18. Ma YC, Zuo L, Chen JH, et al. Modified glomerular filtration rate estimating equation for Chinese patients with chronic kidney disease. J Am Soc Nephrol. 2006 Oct;17(10):2937-44

19. Huang J, Liu G, Zhang YM, et al. Plasma soluble urokinase receptor levels are increased but do not distinguish primary from secondary focal segmental glomerulosclerosis. Kidney Int. 2013 Aug;84(2):366-72.

20. Qu Z, Cui Z, Liu G, et al. The distribution of lgG subclass deposition on renal tissues from patients with anti-glomerular basement membrane disease. BMC Immunol. 2013 Apr 15;14:19.

21. Wang J, Cui Z, Lu J, et al. Circulating antibodies against thrombospondin type-l domain-containing 7A in Chinese patients with idiopathic membranous nephropathy. Clin J Am Soc Nephrol. 2017 Oct;12(10):1642-51.
22. Disease K. Improving Global Outcomes (KDIGO) Glomerulonephritis Work Group. KDIGO clinical practice guideline for glomerulonephritis. Kidney Inter, Suppl. 2012;2:139-274.

23. Cattran DC, Pei Y, Greenwood CM, et al. Validation of a predictive model of idiopathic membranous nephropathy: its clinical and research implications. Kidney Int. 1997 Mar;51(3):901-7.

24. van den Brand JA, Hofstra JM, Wetzels JF. Prognostic value of risk score and urinary markers in idiopathic membranous nephropathy. Clin J Am Soc Nephrol. 2012 Aug;7(8):1242-8.

25. Marx BE, Marx M. Prediction in idiopathic membranous nephropathy. Kidney Int. 1999 Aug:56(2):666-73.

26. Reichert LJ, Koene RA, Wetzels JF. Prognostic factors in idiopathic membranous nephropathy. Am J Kidney Dis. 1998 Jan;31(1):1-11.

27. Shiiki H, Saito T, Nishitani $Y$, et al. Prognosis and risk factors for idiopathic membranous nephropathy with nephrotic syndrome in Japan. Kidney Int. 2004 Apr;65(4):1400-7.

28. Zhang $\mathrm{BO}$, Cheng $\mathrm{M}$, Yang $\mathrm{M}$, et al. Analysis of the prognostic risk factors of idiopathic membranous nephropathy using a new surrogate end-point. Biomed Rep. 2016 Feb;4(2):147-52.

29. Chen $Y$, Tang $L$, Feng $Z$, et al. Pathological predictors of renal outcomes in nephrotic idiopathic membranous nephropathy with decreased renal function. J Nephrol. 2014 Jun;27(3):307-16.

30. Wu Q, Jinde K, Nishina $M$, et al. Analysis of prognostic predictors in idiopathic membranous nephropathy. Am J Kidney Dis. 2001 Feb;37(2):380-7.

31. Yoshimoto K, Yokoyama H, Wada T, et al. Pathologic findings of initial biopsies reflect the outcomes of membranous nephropathy. Kidney Int 2004 Jan;65(1):148-53.

32. Dumoulin A, Hill GS, Montseny JJ, et al. Clinical and morphological prognostic factors in membranous nephropathy: significance of focal segmental glomerulosclerosis. Am J Kidney Dis. 2003 Jan;41(1):38-48.

33. Becker GJ, Hewitson TD. The role of tubulointerstitial injury in chronic renal failure. Curr Opin Nephrol Hypertens. 2000 Mar;9(2):133-8.

34. Ruggenenti P, Debiec H, Ruggiero B, et al. Anti-phospholipase A2 receptor antibody titer predicts post-rituximab outcome of membranous nephropathy. J Am Soc Nephrol. 2015 Oct;26(10):2545-58.

35. Hoxha E, Thiele I, Zahner G, et al. Phospholipase A2 receptor autoantibodies and clinical outcome in patients with primary membranous nephropathy. J Am Soc Nephrol. 2014 Jun;25(6):1357-66
Ready to submit your research? Choose BMC and benefit from:

- fast, convenient online submission

- thorough peer review by experienced researchers in your field

- rapid publication on acceptance

- support for research data, including large and complex data types

- gold Open Access which fosters wider collaboration and increased citations

- maximum visibility for your research: over $100 \mathrm{M}$ website views per year

At $\mathrm{BMC}$, research is always in progress.

Learn more biomedcentral.com/submissions 